\title{
Performance of Silicon Pixel Detectors at Small Track Incidence Angles for the ATLAS Inner Tracker Upgrade
}

\author{
Simon Viel ${ }^{\mathrm{a}, *}$, Swagato Banerjee ${ }^{\mathrm{b}, 1}$, Gerhard Brandt ${ }^{\mathrm{a}, 2}$, Rebecca Carney ${ }^{\mathrm{a}}$, Maurice Garcia-Sciveres ${ }^{\mathrm{a}}$, \\ Andrew Straiton Hard ${ }^{\mathrm{b}}$, Laser Seymour Kaplan ${ }^{\mathrm{b}}$, Lashkar Kashif ${ }^{\mathrm{b}}$, Aliaksandr Pranko ${ }^{\mathrm{a}}$, Julia Rieger ${ }^{\mathrm{a}, \mathrm{c}, 2}$, \\ Julian Wolf ${ }^{\mathrm{a}, 3}$, Sau Lan $\mathrm{Wu}^{\mathrm{b}}$, Hongtao Yang ${ }^{\mathrm{b}}$ \\ ${ }^{a}$ Physics Division, Lawrence Berkeley National Laboratory and University of California, Berkeley CA, United States of America \\ ${ }^{b}$ Department of Physics, University of Wisconsin, Madison WI, United States of America \\ ${ }^{c}$ II Physikalisches Institut, Georg-August-Universität, Göttingen, Germany
}

\begin{abstract}
In order to enable the ATLAS experiment to successfully track charged particles produced in high-energy collisions at the High-Luminosity Large Hadron Collider, the current ATLAS Inner Detector will be replaced by the Inner Tracker (ITk), entirely composed of silicon pixel and strip detectors. An extension of the tracking coverage of the ITk to very forward pseudorapidity values is proposed, using pixel modules placed in a long cylindrical layer around the beam pipe. The measurement of long pixel clusters, detected when charged particles cross the silicon sensor at small incidence angles, has potential to significantly improve the tracking efficiency, fake track rejection, and resolution of the ITk in the very forward region. The performance of state-of-the-art pixel modules at small track incidence angles is studied using test beam data collected at SLAC and CERN.
\end{abstract}

Keywords: LHC, High Luminosity, ATLAS Upgrade, Inner Tracker, Pixel Detector, Forward Tracking

\section{Introduction}

The Large Hadron Collider (LHC) is foreseen to be upgraded in 2024-2026 to become the High-Luminosity LHC, delivering proton-proton collisions at a levelled luminosity of $7.5 \times 10^{34} \mathrm{~cm}^{-2} \mathrm{~s}^{-1}$, with an average pileup of $\langle\mu\rangle=200$ collisions every 25 ns. The ATLAS experiment [1] will need to be upgraded to successfully operate given such high collision rates. In particular, the current ATLAS Inner Detector will need to be completely replaced by a new Inner Tracker (ITk), made of silicon pixel and strip detectors.

Different design layouts for the ITk are currently being evaluated using simulated data, based on tracking performance figures of merit such as tracking efficiency, resolution, and pattern recognition capabilities. Many of these designs aim to extend the tracking coverage of the ITk to very forward pseudorapidity ${ }^{4}$ values up to $|\eta|=4$, in order

\footnotetext{
* Corresponding author.

Email address: sviel@lbl.gov (Simon Viel)

${ }^{1}$ Present address: Department of Physics \& Astronomy, University of Louisville, Louisville KY, United States of America

${ }^{2}$ Present address: II Physikalisches Institut, Georg-AugustUniversität, Göttingen, Germany

${ }^{3}$ Permanent address: Department of Physics, McGill University, Montréal QC, Canada

${ }^{4}$ ATLAS uses a right-handed coordinate system with the $z$-axis along the beam pipe. Cylindrical coordinates $(r, \phi)$ are used in the transverse plane. Pseudorapidity is defined in terms of the polar angle $\theta$ as $\eta=-\ln \tan (\theta / 2)$.
}

to enable significant improvements in pileup jet suppression, vertex reconstruction, $b$-tagging, lepton identification and missing transverse momentum resolution [2]. Sensors placed parallel to the beam pipe at very forward pseudorapidity are crossed by tracks at small incidence angles.

The goal of this study is to characterize the performance of pixel detectors at incidence angles from 2 to 15 degrees with respect to the sensor plane, using test beam data collected in 2015 at SLAC End Station A and at the CERN SPS. Following more information about the context relevant to this research in Section 2, the devices under test, experimental setup and data analysis methods are described in Section 3. Results from the SLAC and CERN test beams are presented in Sections 4 and 5 respectively.

\section{Extended Inner Pixel Barrel Layers for the ITk}

A possible ITk layout design currently under investigation is shown in Figure 1. Hermetic coverage up to $|\eta|=4$ is achieved by means of an extension of the two inner pixel barrel layers up to $z=1.22 \mathrm{~m}$, accompanied by an adequate number of pixel ring layers in the forward region.

With this layout, the innermost pixel barrel layer provides a measurement as close as possible to the interaction point for the entire pseudorapidity range, resulting in expected improvements in tracking efficiency and resolution. Charged particle tracks at high pseudorapidity cross the extended pixel barrel layers at small incidence angles, giving rise to long clusters of pixel hits, as shown in Figure 2. 


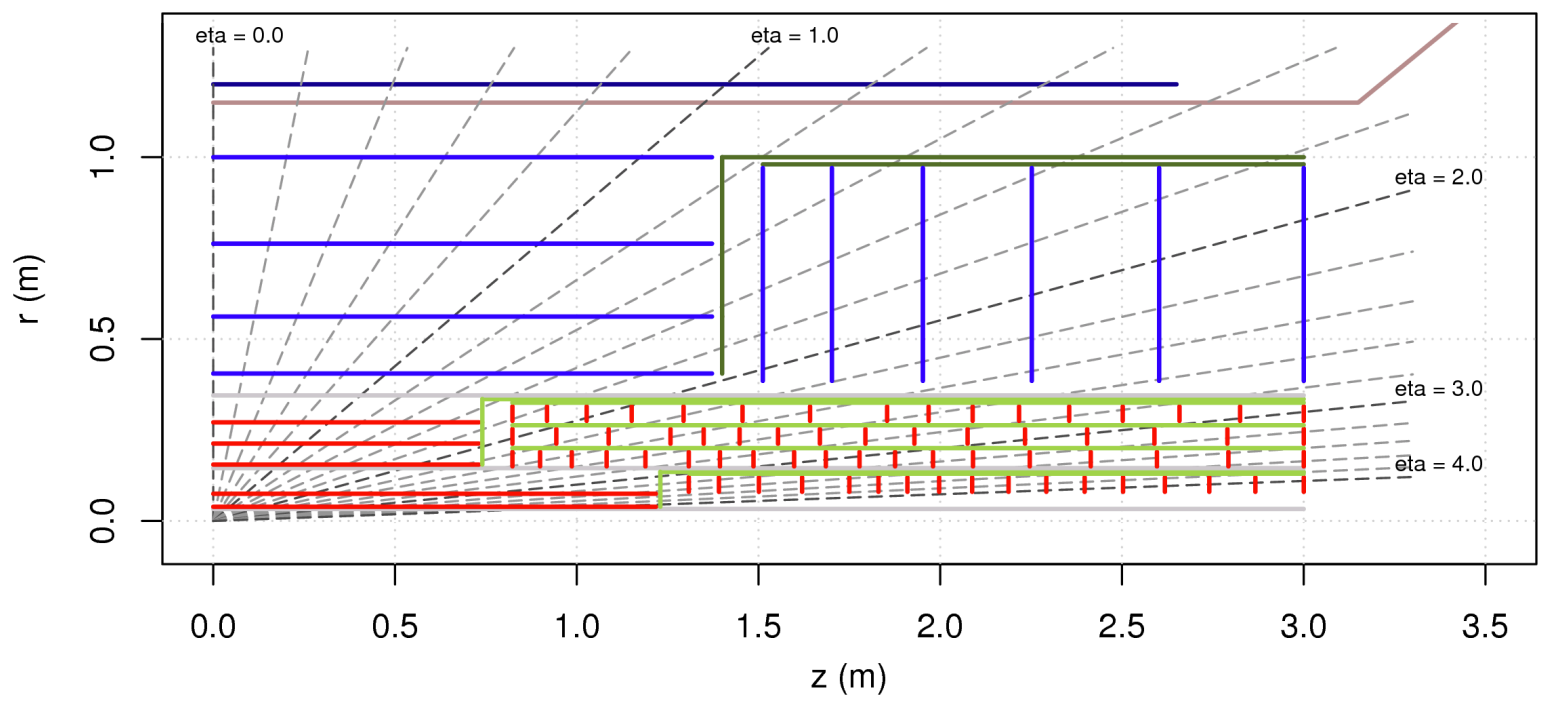

Figure 1: Possible ITk layout design with pseudorapidity coverage up to $|\eta|=4$. Stereo strip detector layers are shown in light blue, and the pixel detector is shown in red. A possible routing for services is shown in green. The brown line outside the ITk volume represents the cryostat boundary, and the dark blue line represents the coil of the solenoid magnet. Lines of constant pseudorapidity are shown in grey.

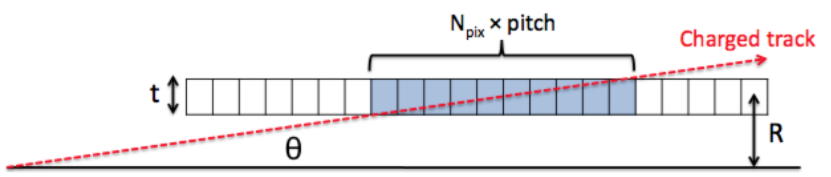

LHC beam line

Figure 2: Schematic of a long pixel cluster caused by the passage of a charged particle with a small track incidence angle $\theta$.

Measured long clusters can be considered as short track segments, or "tracklets", since they contain directional information about the track. Explicitly, for a given sensor thickness $t$ and pitch $p$, the cluster length $N_{\text {pix }}$ provides a measurement of the track angle $\theta$ as

$$
\tan \theta=\frac{t}{\left(N_{\text {pix }}-\delta\right) \times p}
$$

where $\delta=1$ is used to account for the difference between the measured cluster length and the distance between the track entry and exit points in the beam direction.

It is therefore possible to use long clusters as seeds during the pattern recognition phase of track reconstruction, by only considering track candidates where the pixel cluster lengths are compatible with the track angle. This is expected to result in considerable savings in computing time, as well as lower fake track rates, compared with a detector where this information is absent. Early simulation results show that the cluster length information can be used for pattern recognition as soon as the expected cluster length is greater than or equal to 3 pixels.

Pixel modules in the ITk are currently expected to have a pitch of $50 \mu \mathrm{m} \times 50 \mu \mathrm{m}$, and a sensor thickness of 100 to $150 \mu \mathrm{m}$. With a sensor thickness of $150 \mu \mathrm{m}$, clusters are expected to have a length of 3 pixels for tracks at $|\eta|=0.63,18$ pixels at $|\eta|=2.5$ and 83 pixels at $|\eta|=4.0$.
In the absence of multiple scattering effects and without making use of pixel charge information, the resolution on the cluster length $N_{\text {pix }}$ is limited by the pitch, in pixel units $1 / \sqrt{12}$ for each of the entry and exit pixel positions, for a combined total of $1 / \sqrt{6}$. Following Equation 1, the angular resolution achieved with this technique therefore improves for smaller values of the track incidence angle $\theta$. As will be seen, the influence of multiple scattering can be appreciated only for the smallest angle $\left(2^{\circ}\right)$, indicating that for $50 \mu \mathrm{m} \times 50 \mu \mathrm{m}$ pixels, multiple scattering does not degrade the measurement until the path length in silicon exceeds $3 \mathrm{~mm}$. This is consistent with analytic calculation.

\section{Experimental Methods}

\subsection{Devices Under Test}

Four un-irradiated pixel modules, produced for the Insertable B-Layer (IBL) [3] upgrade to the ATLAS detector, are tested: two double-chip modules with planar sensors (200 $\mu \mathrm{m}$ thick), with production numbers 93-04-03 and 94-01-04 and two single-chip modules with 3D sensors (230 $\mu \mathrm{m}$ thick), named 22-08-25 and ATLAS09 FBK12. Each sensor is bump-bonded to the corresponding number of FE-I4 readout chips, also developed for the IBL.

FE-I4 readout chips have 80 columns by 336 rows of $250 \mu \mathrm{m} \times 50 \mu \mathrm{m}$ pixel cells. Each cell amplifies the collected signal and compares it to a programmable threshold. While for the devices under test the minimal threshold is $1000 \mathrm{e}^{-}$, data were also collected at thresholds of $2000 \mathrm{e}^{-}$ and $3000 \mathrm{e}^{-}$. In contrast, ITk modules are expected to have a minimal discriminator threshold of $600 \mathrm{e}^{-}$.

The time-over-threshold (ToT) is measured in units of the 25 ns clock signal. For each threshold value of interest, the devices under test were tuned to yield a ToT value 
of 10 units for a $16 \mathrm{ke}^{-}$signal. Noisy and malfunctioning pixels were masked during this process: the resulting noise rates as measured with a dedicated scan were $<1$ hit per minute in any given module.

In addition to testing the pixel modules at different incidence angles and threshold values, the sensors were tested in a range of reverse bias voltage values. Planar modules were operated fully depleted at $80 \mathrm{~V}$ or $120 \mathrm{~V}$, while 3D modules require lower values: module 22-08-25 was operated most often at $9 \mathrm{~V}$ with a few runs at $2 \mathrm{~V}$, and module ATLAS09 FBK12 was tested in a range from $2 \mathrm{~V}$ to 40 V. Except where discussed in Section 5, no difference in performance is observed for these bias voltage variations.

\subsection{Test Beam Setup}

A first beam test was performed at SLAC in April and May 2015 with $10 \mathrm{GeV}$ electrons, incident on the devices in few-particle bunches at a rate of $5 \mathrm{~Hz}$. Modules were mounted on carbon plates, and then placed on an aluminum rig adjustable to pre-calibrated angular positions at $2^{\circ}, 4^{\circ}, 6^{\circ}, 10^{\circ}$ and $15^{\circ}$ with respect to the horizontal, such that to first order the angle between the beam line and each sensor plane takes one of these values. Two or three modules were simultaneously operated in each run, with modules in the short pixel direction first in the beam. The modules were placed with the beam either in the long or the short pixel direction, that is with the beam perpendicular to the $50 \mu \mathrm{m}$ pitch or $250 \mu \mathrm{m}$ pitch direction respectively, and were lined up so that most electrons would cross all modules present. No telescope was used, as the long clusters provide sufficient track information.

Module ATLAS09 FBK12 was then sent to the CERN SPS test beam facility for operation with a $180 \mathrm{GeV}$ pion beam in May 2015. There, a telescope consisting of 6 FE-I4 DC modules was used, with the device under test placed between two groups of three pixel detector planes each, with the telescope modules perpendicular to the beam. The same carbon mounting plate was used, on a different positioning device designed to be placed between the arms of the telescope. Data were taken systematically at angles of $2^{\circ}, 4^{\circ}, 6^{\circ}, 10^{\circ}$ and $15^{\circ}$ measured between the carbon plate and the beam line, in both the long and short pixel directions, for thresholds of $1000 \mathrm{e}^{-}$and $2000 \mathrm{e}^{-}$, and reverse bias voltages of $2,4,6,8,10,30$ and $40 \mathrm{~V}$.

\subsection{Analysis Methods}

During data taking, all pixel hits registered in 16 consecutive periods of $25 \mathrm{~ns}$ each following the reception of an external trigger are considered part of the same event. In each event, clusters are formed from adjacent hits on a same module, allowing for a maximum of 10 (1) consecutive holes in the short (long) pixel direction. Masked pixels are taken into account during cluster reconstruction, and are not counted as pixel holes.

Figure 3 shows an example cluster length distribution observed with a module in the short pixel direction. The beam incidence angle is measured from this distribution using a Gaussian fit to the signal peak: the mean angle is calculated from the fitted mean cluster length using Equation 1, and the angular resolution is obtained from the standard deviation. Off-peak clusters mainly come from secondary particles and from clusters truncated by the chip edges, and are not considered further. Systematic effects due to the choice of values for the number of consecutive pixel holes allowed during reconstruction, for initializing the Gaussian fits, and for $\delta$ in Equation 1 are neglected in the following sections.

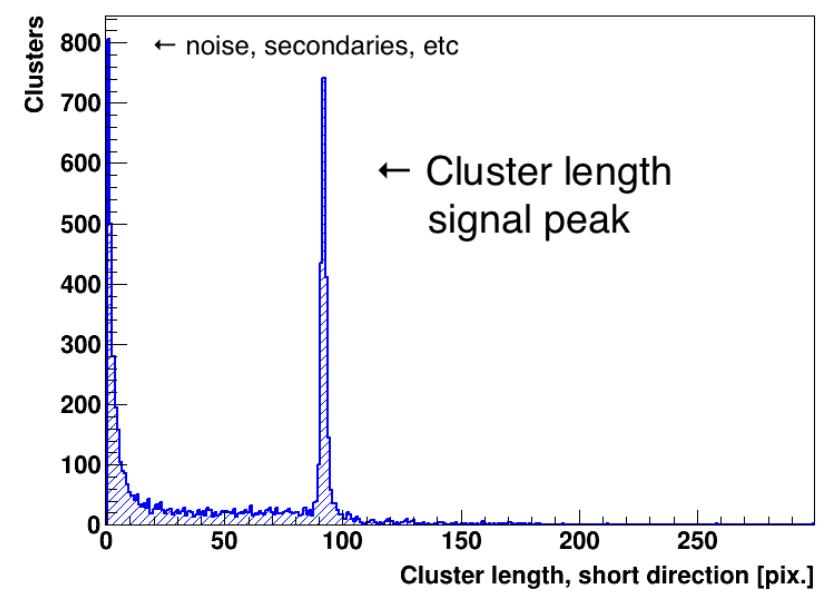

Figure 3: Example cluster length distribution from the SLAC dataset: module 94-01-04 placed in the short pixel direction, operated with $120 \mathrm{~V}$ reverse bias and a threshold of $1000 \mathrm{e}^{-}$. Using this distribution the beam incidence angle is measured to be $2.5^{\circ}$.

Signal clusters are then trimmed to remove delta rays using the following algorithm: clusters are considered as neighboring lines, along chip columns or rows depending on the beam direction. Lines with consecutive holes larger than the length allowed at the clustering step are removed, as are lines not meeting a minimum length requirement depending on the beam direction and angle. The resulting trimmed long clusters are one or two pixels wide.

The pixel hit efficiency is calculated using trimmed clusters, by counting the fraction of pixels hit between the first and the last hits of each cluster. Because the timing window used in this study is wider than a single period of $25 \mathrm{~ns}$, as is the case during the operation of the ATLAS detector, the efficiency values reported here are generally higher than the in-time efficiency. The timing distributions are narrow, suggesting that this correction is small.

In the CERN dataset, the measurement of tracks using the FE-I4 telescope enables further analysis. Tracking is performed with the telescope data using the Judith software [4], which also outputs alignment constants for the device under test using the unbiased residuals calculated with the center-of-charge positions of the long clusters measured. The telescope resolution at the device under test is $14 \mu \mathrm{m}$ in the horizontal direction and $8.5 \mu \mathrm{m}$ in the vertical direction relative to the experimental hall. 


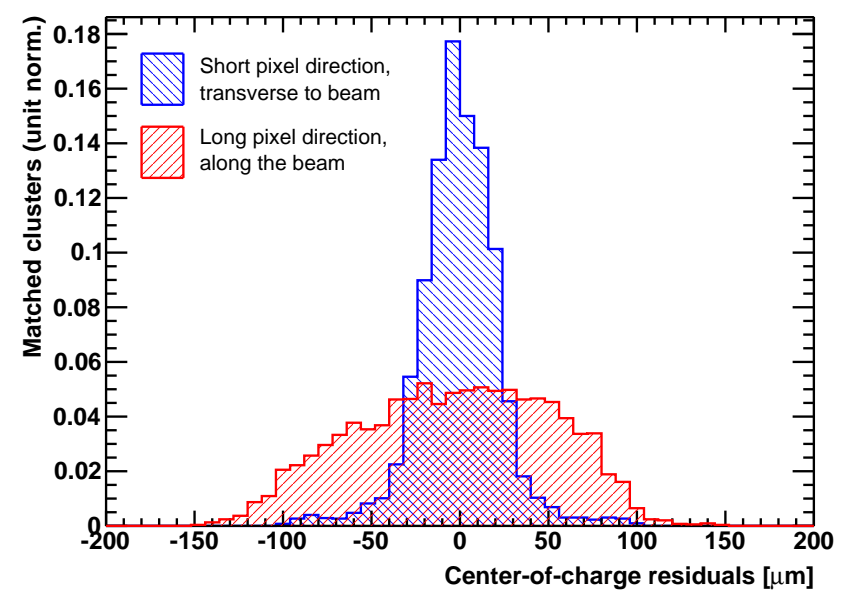

Figure 4: Center-of-charge unbiased residuals, for module ATLAS09 FBK12 at CERN, in the short pixel direction oriented transverse to the beam (blue) and in the long pixel direction along the beam (red).

Figure 4 shows example distributions of unbiased residuals after alignment, for the horizontal and vertical cluster center-of-charge positions measured by module ATLAS09 FBK12. In this run, the module was operated with $40 \mathrm{~V}$ reverse bias and a threshold of $1000 \mathrm{e}^{-}$, and rotated along the vertical axis such that the beam was incident in the long pixel direction with an angle of $1.4^{\circ}$ as measured using the observed cluster length distribution. The root mean square of the distribution is $54 \mu \mathrm{m}$ in the horizontal direction (corresponding to the long pixel direction), and $23 \mu \mathrm{m}$ in the vertical direction (short pixel direction). These residual distributions are measured to be similar for other values of reverse bias voltage and threshold.

Data runs with the configuration described above are of special interest as they allow for in-pixel measurements of charge collection and charge sharing as a function of the intersection of the track with each pixel. Specifically, for each pixel crossed by a track, a local coordinate system is defined with $X$ in the long pixel direction, $Y$ in the short pixel direction, and $Z$ in the sensor depth direction. The intersection point of the track with the $(Y, Z)$ plane at the middle of the pixel in $X$ is calculated.

For the charge collection measurement, the ToT value measured by each pixel hit in trimmed clusters is recorded as a function of the local position of this intersection point. The mean ToT observed in each run is calculated in bins $5 \mu \mathrm{m}$ wide separately as a function of the position $Y$ and the depth $Z$. The charge sharing probability $\left(P_{S h}\right)$ is calculated as a function of the in-pixel $Y$ position where the track crossed the sensor for each trimmed cluster, by comparing the number of hits in the main row $\left(N_{m}\right)$ to the number of hits in the neighboring row $\left(N_{n}<N_{m}\right)$ :

$$
P_{S h}=1-\frac{N_{m}-N_{n}}{N_{m}+N_{n}}
$$
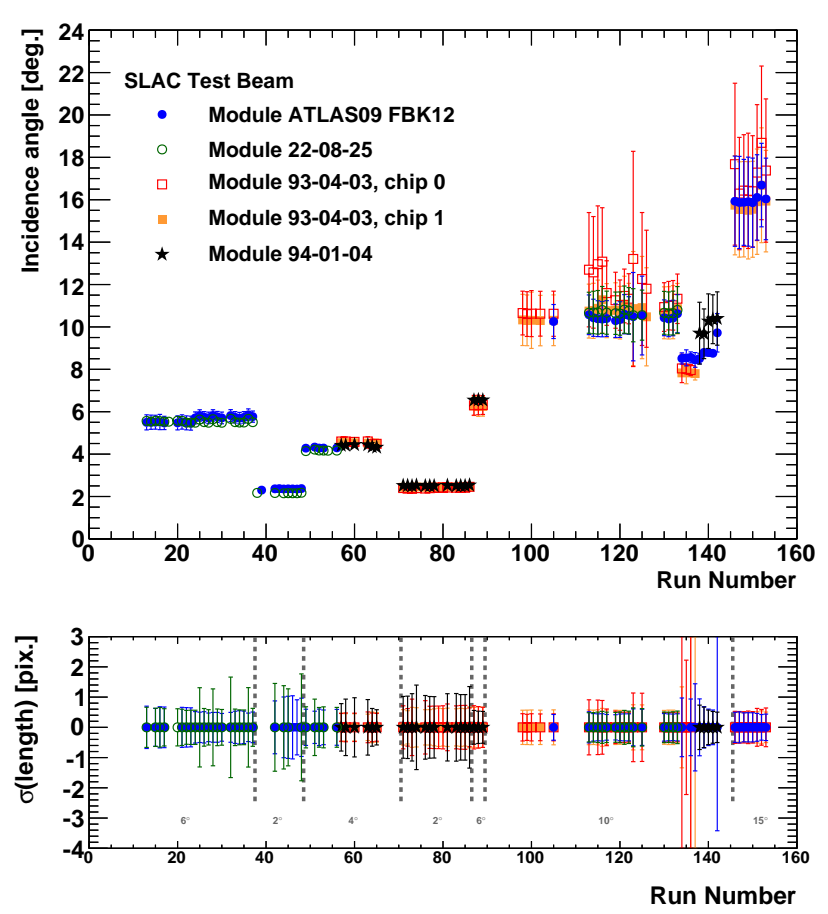

Figure 5: Incidence angle measurements from the SLAC dataset. The bottom inset shows the cluster length resolution from each run.

\section{SLAC Test Beam Results}

Incidence angles measured using the cluster length technique are shown for each run from the dataset collected at SLAC in Figure 5. The sensor planes are not exactly parallel to the rig, which explains why the angles measured between the sensors and the beam may differ from the rig's angle with respect to the horizontal. An excellent angular resolution is observed with all modules, especially at the lowest incidence angles, e.g. $1 \%$ resolution for $\theta \sim 2^{\circ}$. In most cases this resolution is limited by the sensor pitch, except for runs at $2^{\circ}$ where small scattering effects are starting to be visible.

Figure 6 shows the pixel hit efficiency measured in the short pixel direction. Modules operated at a threshold of $1000 \mathrm{e}^{-}$have a very good hit efficiency: almost perfect for planar modules and above $93 \%$ for 3D modules at all values of incidence angle and reverse bias voltage. At higher thresholds the efficiency is lower, confirming the need for ITk modules to operate at as low a threshold as possible. The efficiency measured in the long pixel direction is above $99 \%$ for all modules in all tested operating conditions.

\section{CERN Test Beam Results}

In the CERN dataset, the pixel hit efficiency measured for module ATLAS09 FBK12 in the long pixel direction is above $99.9 \%$ for all values of threshold, reverse bias voltage and incidence angle. The hit efficiency measured in the short pixel direction is shown in Figure 7. Here inefficiencies are observed at small bias voltage values, and more 


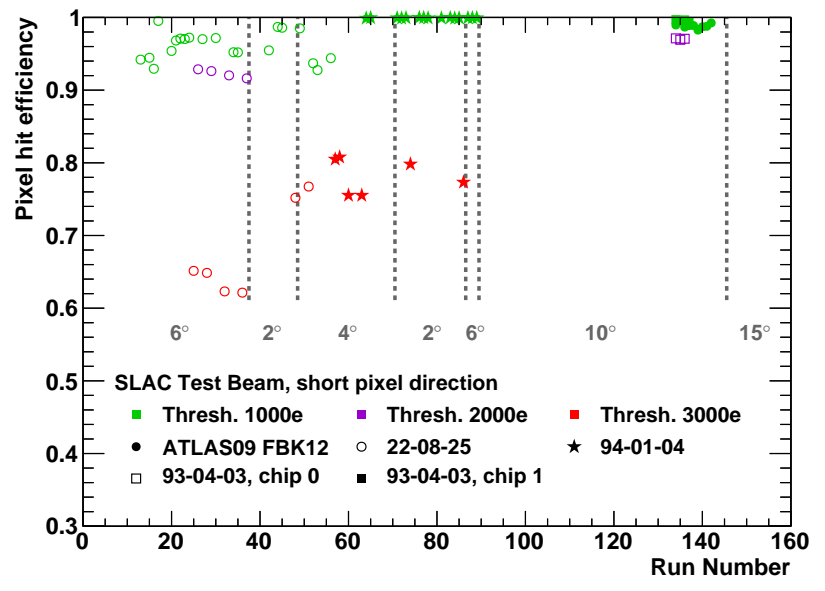

Figure 6: Pixel hit efficiency in the short pixel direction from the SLAC dataset.

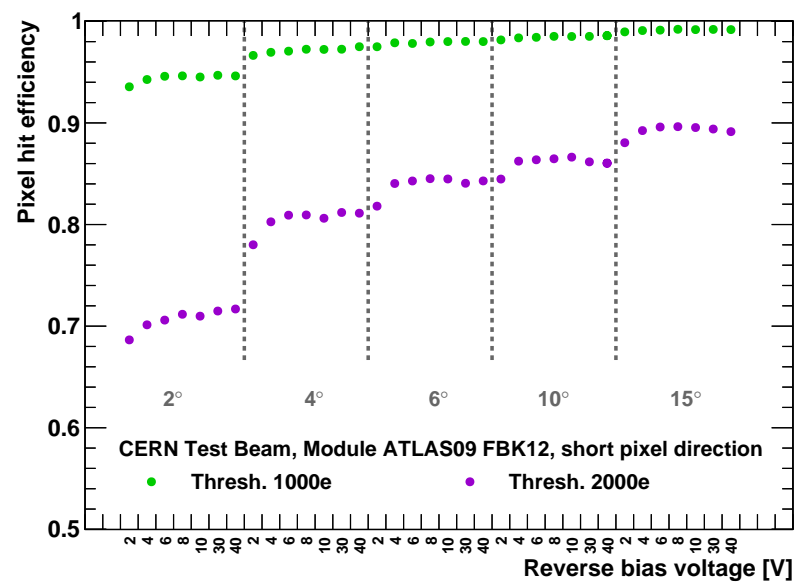

Figure 7: Pixel hit efficiency in the short pixel direction measured in the CERN dataset.

importantly the hit efficiency decreases with the track incidence angle: at $2^{\circ}$ it lowers to $95 \%$ (71\%) for high bias voltage at a threshold of $1000 \mathrm{e}^{-}\left(2000 \mathrm{e}^{-}\right)$. Further studies including in-pixel efficiency measurements would be helpful to understand the hit efficiency dependence on the incidence angle. This effect has a minimal impact on the cluster reconstruction efficiency because of the large number of allowed consecutive holes in the short pixel direction.

Figure 8 shows the incidence angles measured in the CERN dataset. A small bias is seen at high threshold values: the charge deposition in the entry and exit pixels of the track is more likely to be below threshold, and thus clusters may appear shorter than they should be, resulting in slightly higher values for the measured angle. This systematic bias could be corrected by adjusting the value of $\delta$ in Equation 1 for different thresholds. In other respects the resolution as indicated by the Gaussian standard deviation is again excellent, limited by pixel pitch except for the small multiple scattering effects seen at $2^{\circ}$ in the short pixel direction.
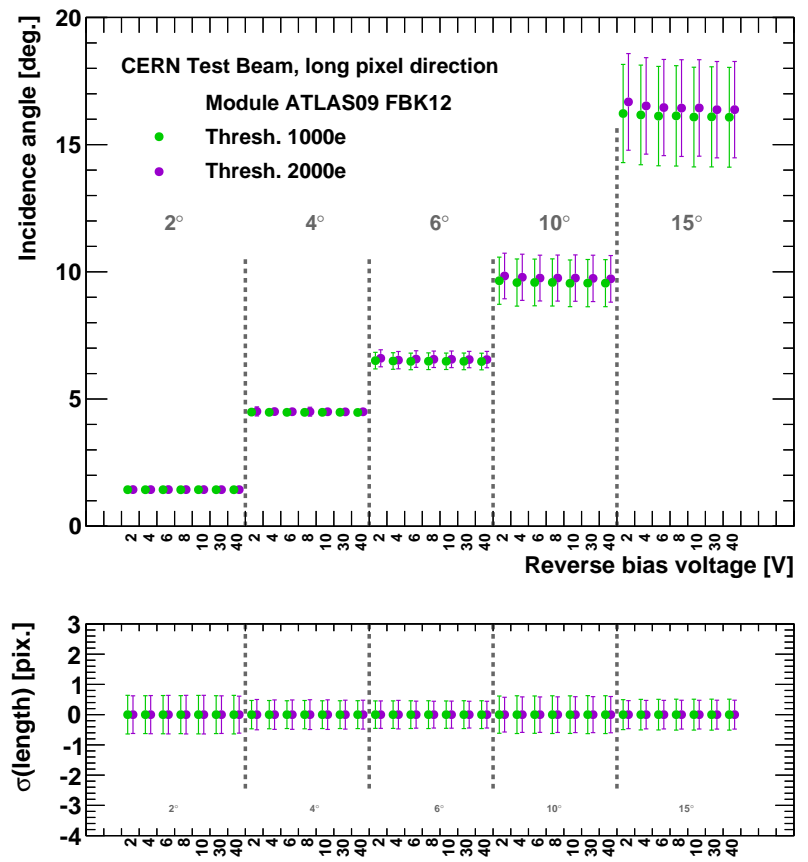

Reverse bias voltage [V]
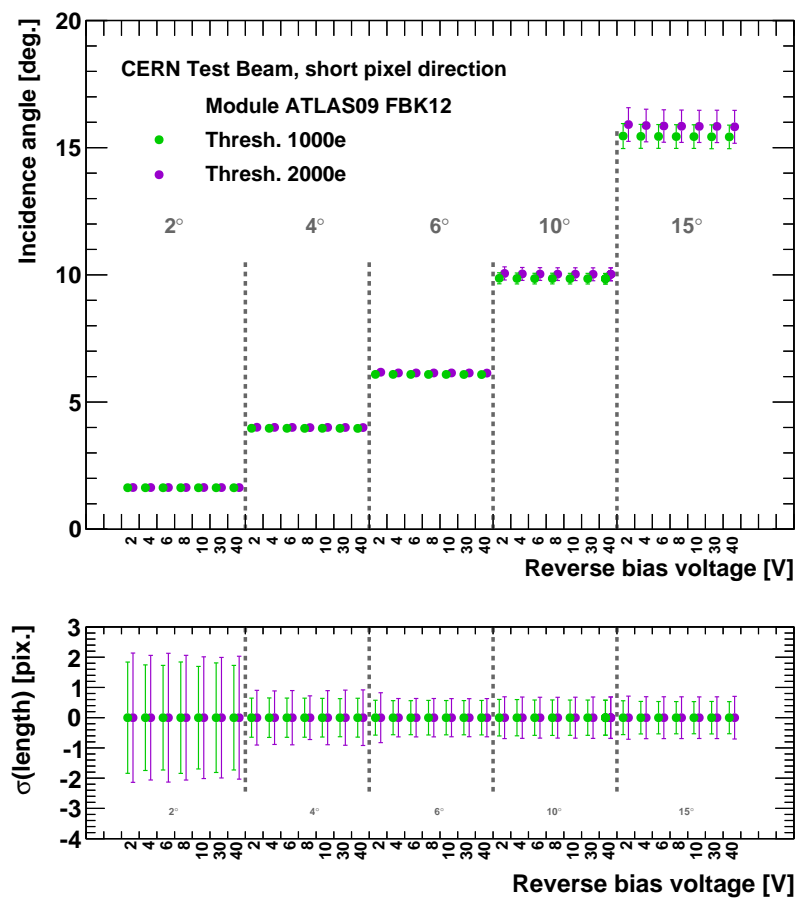

Figure 8: Incidence angle measurements in the long pixel direction (top) and short pixel direction (bottom) from the CERN dataset. The bottom inset shows the cluster length resolution from each run.

The results of the charge collection measurement are shown in Figure 9, and charge sharing probabilities are presented in Figure 10. As expected, less charge collection is observed close to the edge of the sensor, that is in the region where charge sharing occurs, and both effects are stronger at lower reverse bias voltage values. No effect is observed as a function of depth except at the sensor edges, 

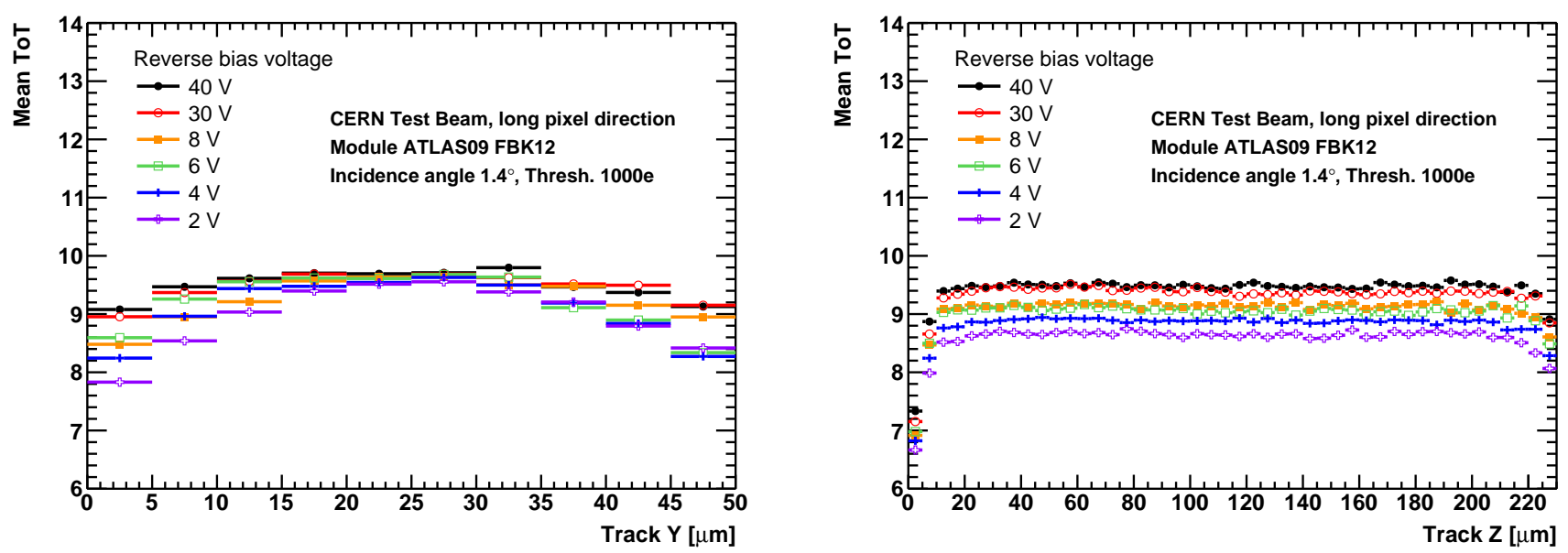

Figure 9: In-pixel mean ToT measured as a function of the incident track position in the short pixel direction (left) and the sensor depth direction (right), for different bias voltage values applied to module ATLAS09 FBK12 at CERN.

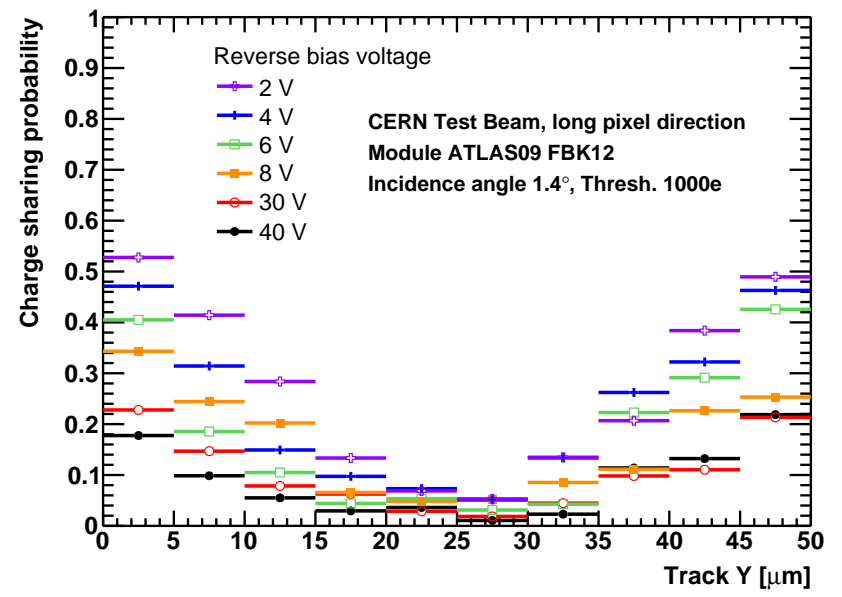

Figure 10: In-pixel charge sharing probability measured as a function of the incident track position in the short pixel direction, for different bias voltage values applied to module ATLAS09 FBK12 at CERN.

where charge collection is lower than in the bulk of the sensor. At a threshold of $2000 \mathrm{e}^{-}$, charge collection results are similar, while the measured charge sharing probabilities are lower by approximately $25 \%$ in relative terms. This observation is consistent with the shared charge being less frequently sufficient to reach the higher threshold.

\section{Conclusion}

Test beam results with un-irradiated ATLAS IBL planar and 3D modules demonstrate the excellent performance of these devices at small track incidence angles. Long pixel clusters are reconstructed with high efficiency by modules operated at low threshold values, and outstanding tracking angular resolution is achieved using the cluster length information. This constitutes the first proof of concept for the ITk layout design with extended inner pixel barrel layers.
The most important next step will be to evaluate the performance at small track incidence angles of sensors irradiated at the levels expected during operation at the High-Luminosity LHC, including an evaluation of the intime efficiency as a function of threshold, bias voltage and incidence angle. Measurements with new prototype ITk modules including readout chips developed by the RD53 collaboration will also be essential. Alongside analyses with simulated data, test beam studies using the methods presented here will continue to provide critical input toward completing the design of the ATLAS ITk upgrade.

\section{Acknowledgements}

We thank CERN and SLAC for the very successful operation of their test beam facilities, with special thanks to Carsten Hast (SLAC) and Mathieu Benoit (Université de Genève) for their support at SLAC and at CERN, respectively. This work was supported by the U.S. Department of Energy under contract numbers DE-AC02-05CH11231, DE-FG02-95ER40896 and DE-AC02-76SF00515. This research used resources of the National Energy Research Scientific Computing Center, a DOE Office of Science User Facility supported by the Office of Science of the U.S. Department of Energy under contract number DE-AC0205CH11231. We gratefully acknowledge additional support from the Alexander von Humboldt Foundation (G. Brandt), from Göttingen International and the Konrad-AdenauerStiftung (J. Rieger), and from the National Sciences and Engineering Research Council of Canada (S. Viel).

\section{References}

\section{References}

[1] ATLAS Collaboration 2008 JINST 3 S08003

[2] ATLAS Collaboration 2015 CERN-LHCC-2015-020

[3] ATLAS Collaboration 2010 ATLAS-TDR-019

[4] G. McGoldrick, M. Červ and A. Gorišek 2014 NIM A 765140 